\title{
Retrospective Analysis of out-of-Hospital Paediatric Intubation by a Dutch Helicopter Emergency Medical Service
}

\author{
Moors Xavier RJ, $M D^{1,2,}$, Oude Alink Michelle B, $M D^{1}$, Bouman Stef JM, $M D^{3}$, Den Hartog Dennis, $M D$, \\ PhD ${ }^{4}$, Houmes Robert Jan, MD, PhD ${ }^{2,5}$ and Stolker Robert Jan, MD, PhD ${ }^{1}$ \\ ${ }^{1}$ Department of Anesthesiology, Sophia Children's Hospital, Erasmus University Medical Center, The Netherlands \\ ${ }^{2}$ HEMS, Erasmus University Medical Center, The Netherlands \\ ${ }^{3}$ Medical Student, Erasmus University Medical Center, The Netherlands \\ ${ }^{4}$ Department of Surgery, Trauma Research Unit, Erasmus University Medical Center, The Netherlands \\ ${ }^{5}$ Department of Pediatric Intensive Care, Erasmus University Medical Center, The Netherlands
}

\begin{abstract}
Background: Endotracheal intubation (ETI) is frequently performed in critically ill or severely injured paediatric patients and remains the gold standard to secure the airway.

Objective: The objective of this retrospective study was to examine the first attempt and overall success rate of out-of-hospital emergency paediatric intubation by a physician-staffed Helicopter Emergency Medicine Service (HEMS).

Methods: This was a retrospective database analysis of all paediatric $(<18$-years) intubations performed by HEMS physicians during a six-year period (2012-2017). The recorded findings included patient demographics, operator background, airway interventions (including intubation attempts), video-assisted intubation, and complications.

Results: Three hundred and sixty-one paediatric patients required the airway to be secured. In 280 of these cases, a HEMS physician performed the intubation. The first attempt success rate was $227(81 \%)$ and the overall ETI success rate was $276(98.5 \%)$ after one or more intubation attempts. Surgery was necessary to clear the airway in only one case.

Conclusion: Prehospital ETI of critically injured or ill paediatric patients had a high success rate when performed by HEMS physicians in The Netherlands. In paediatric cases with a potentially compromised airway, the threshold for HEMS deployment should be low.
\end{abstract}

\section{Keywords}

HEMS, Paediatric, Intubation, Prehospital

\section{Introduction}

Little is known about the success rate and complications of paediatric endotracheal intubation (ETI) in a prehospital setting by a Helicopter Emergency Medical Service (HEMS). The Rotterdam HEMS provides care for patients of all ages, including paediatric patients. Currently, paediatric patients are involved in $22 \%$ of all calls, which makes it a unique service. These calls are for a wide variety for critical illnesses including sepsis, cardiopulmonary resuscitation, respiratory failure, and trauma. The purpose of airway management is to achieve adequate tissue oxygenation and ventilation, while limiting aspiration. One of the key goals of resuscitation and critical care is to establish and maintain a patent airway by endotracheal intubation [1,2] and remains the standard method to secure the airway [3]. Unique challenges can be seen when intubating a paediatric patient in an out-of-hospital environment [4-7], making

*Corresponding author: Xavier RJ Moors, MD, Department of Anesthesiology, Sophia Children's Hospital, Erasmus University Medical Center, P.O. Box 2060, 3015 CN Rotterdam, The Netherlands, Tel: +31-107036020, Fax: +31-107039698

Accepted: December 28, 2019

Published online: December 30, 2019

Citation: Moors XRJ, Oude Alink MB, Bouman SJM, et al. (2019) Retrospective Analysis of out-of-Hospital Paediatric Intubation by a Dutch Helicopter Emergency Medical Service. Clin Pediatr Res 3(1):56-62 
Citation: Moors XRJ, Oude Alink MB, Bouman SJM, et al. (2019) Retrospective Analysis of out-of-Hospital Paediatric Intubation by a Dutch Helicopter Emergency Medical Service. Clin Pediatr Res 3(1):56-62

it a potentially risky procedure. Rates of successful ETI vary depending on the investigated patient group and the qualifications of the intubating health care provider [8-11]. The ETI success rate for pre-hospital paediatric patients is between 55 and $100 \%$, with a high complication rate varying from unrecognized oesophageal intubation to potentially "lethal" ventilator settings for less experienced emergency medical service (EMS) health care providers [12]. There is evidence in the Netherlands that intubations performed by paramedics are less safe and successful than other, less invasive techniques such as bag-valve-mask ventilation or even laryngeal mask airway placement [3,13-15]. Even intubations performed by physicians are prone to critical errors and failure if they have insufficient training and equipment, and intubation success may depend on physician's speciality [4,16-18]. For out-of-hospital paediatric ETI, success rates are generally lower than for the adult population $[4,19]$. Due to a lack of frequent exposure to paediatric patients and paediatric intubations, poor performances are seen for all providers of emergency medical care $[14,15,20]$. In addition, frequent training is needed to maintain adequate airway management, because these skills rapidly deteriorate after training, making it challenging given limited resources and competing needs for training on other topics [21]. There are some reports of pre-hospital ETI success rates comparable to the in-hospital rates, especially if performed by highly skilled physicians $[22,23]$. Successful airway management requires training, skills, and ongoing experience to consistently perform these procedures in an effective, timely, and safe manner [12]. Many EMS regions in The Netherlands consider ETI the gold standard for paediatric airway management; while, others have abandoned ETI due to safety concerns, highlighting the controversy among experts in out-of-hospital care [13-15]. Analyses of such findings have led authors to suggest restricting (paediatric) intubation to physicians alone $[15,24]$.

The primary endpoints of this study were the first attempt and overall success rates for out-of-hospital, at the scene paediatric ETI by physician-led HEMS. Secondary endpoints were the type of intubation (direct laryngoscopy or video-assisted intubation using the McGrath video laryngoscope); complications of airway management, including failure of intubation; success by operator type; patient's age, and medication-assisted intubation.

\section{Materials and Methods}

\section{Study design}

We performed a retrospective analysis during a sixyear period (2012-2017) of a custom-made TRIN database that included all consecutive deployments by the Rotterdam HEMS. This database also contained findings about paediatric ETI, the number of ETI attempts, success rate, medication given prior to intubation, and by whom the intubation was performed (trauma surgeon versus anaesthesiologist). A Microsoft access Query was performed on this TRIN database including patients less than 18-years of age and intubation. Only two physicians abstracted the data from the original HEMS database. The definition of an intubation attempt was the preparation for intubation, with or without medication, and trying to visualize the vocal cords using direct or video assisted laryngoscopy for an ETI. If tube placement was not successful and the (video assisted) laryngoscope was withdrawn from the oropharynx, this was marked as end of the intubation attempt.

Positioning of the tracheal tube into the oesophagus and alternative airway access manoeuvers were scored. Tube position was verified by fogging of the tube during expiration, symmetrical thoracic movements, auscultation of breath sounds bilaterally, depth of tube placement and capnography. In some cases, ultrasound was used to verify movement of the pleura. All paediatric patients intubated in the presence of the HEMS were included. Paediatric patients intubated prior to arrival of the HEMS were excluded because of lack of proper documentation by EMS personnel. We considered $P<0.05$ as statistical significant.

\section{Study setting}

The Netherlands has a population of over 17 million people and covers an area of $42,508 \mathrm{~km}^{2}$. There are four HEMS teams in the Netherlands; hence, the country is divided into four areas. Only a small part of the East and Southeast is served by HEMS from centres in Germany just across the border. In the Netherlands HEMS was introduced in 1995, enabling additional care by a medical team to the regular ambulance crew. A HEMS team consists of a physician (board-certified trauma surgeon or anaesthesiologist), a specialized nurse (registered nurse from the Emergency Department [ED] or paramedic) and a helicopter pilot. Rotterdam HEMS has 11 physicians, nine anaesthesiologists and two trauma surgeons. The group of anaesthesiologists receive surgical skill training (chest drain, surgical airway, thoracotomy, etc.); while, the trauma surgeons receive extended anaesthesia training for two months. During this training, because of the high incidence of paediatric patients, they also receive training in paediatric anaesthesia by our Paediatric Anaesthesia Department for two weeks.

A specially designed emergency vehicle is used if the incident is close by or the weather prevails. According to national protocol by EMS Dispatch HEMS can be dispatched primarily or secondarily by the EMS crew at the incident location. On average it takes around 8 minutes to get to the scene. Sometimes HEMS is first on scene. The HEMS follows the same medical protocols and acts in close collaboration with the EMS. After a few years it became protocol to activate HEMS for stabilizing vitally compromised or seriously injured children because EMS frequently asked HEMS for assisting these children.

\section{Selection of participants}

All children under the age of 18-years on the day of the emergency call were extracted for analysis. From 2012 to 2017, we selected all consecutive patients who required tracheal intubation. After identifying all intubations in paediatric patients, we analysed data regarding the first attempt suc- 
Citation: Moors XRJ, Oude Alink MB, Bouman SJM, et al. (2019) Retrospective Analysis of out-of-Hospital Paediatric Intubation by a Dutch Helicopter Emergency Medical Service. Clin Pediatr Res 3(1):56-62

cess rate, intubation attempts, success by operator type, patient age, medication given prior to intubation, and type of intubation. Inclusion criteria were out-of-hospital paediatric ETI by the Rotterdam HEMS. For the purpose of this study, intubations were classified as with or without medication. The intubation medication includes at least an anaesthetic induction agent and a neuromuscular blockade, sometimes combined with an opioid. The intubations performed without medication occurred during cardiopulmonary arrest. The first-look laryngoscopy was defined as the first passage of the laryngoscope blade between the lips with intention to intubate the trachea, whether or not this led to passage of an endotracheal tube. Maximum efforts were made to complete the dataset. Data not found are reported as unknown in the results. Patients primarily treated in other hospitals and then transferred to our hospital were excluded.

\section{Ethics approval}

The Medical Ethical Committee of the Erasmus University Medical Center (MEC-2018-1376) approved this study.

\section{Results}

In a six-year period (2012-2017), Rotterdam HEMS received 16674 calls in total, of which 7706 (46\%) missions were cancelled by EMS before arriving at the scene. Of the 8968 patients treated, 1905 (21\%) were paediatric patients. In this six-year period, 2609 patients underwent ETI in the prehospital setting, including 361 paediatric patients. Of these paediatric patients, 280 were intubated by a HEMS physician. Due to missing data, only 273 intubations could be analysed (Figure 1). The overall success rate was 276 (98.5\%) and the first attempt success rate was 227 (81\%) (Table 1). There is no significant different in the overall or first attempt success

\section{Total of pediatric HEMS-calls}

$$
\mathrm{n}=1905
$$

Exclusion (1):
- Unknown if ETI-performed:
$n=1$
- No ETI attempted: $\mathrm{n}=1543$

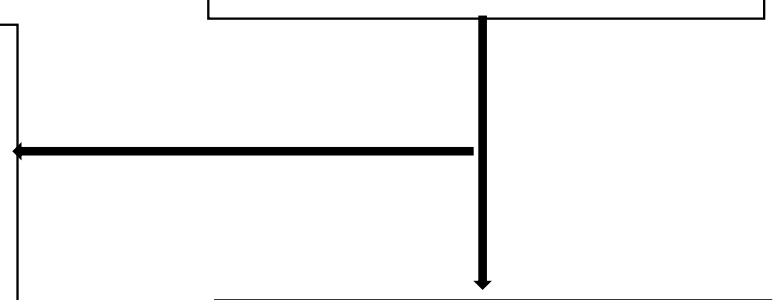

\begin{tabular}{c}
\hline Exclusion (2): \\
- Unknown performer: $\mathrm{n}=3$ \\
- Already intubated in-hospital: \\
$\mathrm{n}=4$ \\
- Other ETI-performer than \\
HEMS-physician: $\mathrm{n}=74$
\end{tabular}

\section{Total of ETI-attempts} performed

$$
\mathrm{n}=361
$$

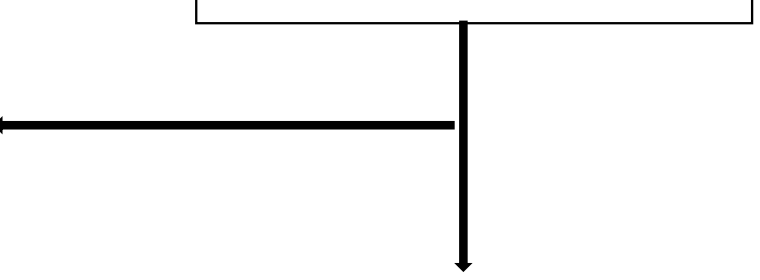

\section{Total of ETI-attempts} performed by HEMS-phycisian

$$
\mathrm{n}=280
$$

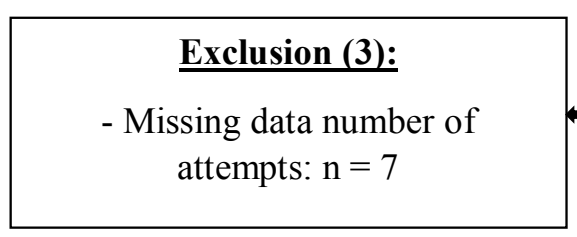

\section{Total of ETI-attempts for analysis}

$$
\mathrm{n}=273
$$

Figure 1: Total intubations with exclusions. 
Citation: Moors XRJ, Oude Alink MB, Bouman SJM, et al. (2019) Retrospective Analysis of out-of-Hospital Paediatric Intubation by a Dutch Helicopter Emergency Medical Service. Clin Pediatr Res 3(1):56-62

Table 1: Number of intubation attempts in relation to overall and first pass success rate.

\begin{tabular}{|l|l|l|l|l|l|l|}
\hline & Number of intubation attempts & $\mathbf{1}^{\text {st }}$ attemp & $\mathbf{2}^{\text {nd }}$ attemp & $\mathbf{3}^{\text {rd }}$ attemp & $\mathbf{4}^{\text {th }}$ attemp & Failed intubation \\
\hline HEMS Physician & 273 & 221 & 41 & 5 & 2 & 4 \\
\hline Overall success rate & & $81 \%$ & $96 \%$ & $97.8 \%$ & $98.5 \%$ & \\
\hline First pass succes rate & & $81 \%$ & & & & \\
\hline
\end{tabular}

Table 2: Classic vs. video assisted intubation.

\begin{tabular}{|l|l|l|l|}
\hline & Classic laryngoscopy & Video assisted laryngoscopy & Unknown device \\
\hline Number off attempts & 174 & 76 & 23 \\
\hline Overall success rate & $173(99.4 \%)$ & $76(100 \%)$ & n.a. \\
\hline First pass success rate & $142(81.6 \%)$ & $61(80.3 \%)$ & n.a. \\
\hline
\end{tabular}

Table 3: Intubation related to age. A: Attempt; O: Overall success; FP: First Pass; F: Failed intubation

\begin{tabular}{|l|l|l|l|l|l|}
\hline Age In years & Number ETI attempts & Overall success rate & First pass & Anesthesiologist A/O/FP/F & Trauma Surgeon A/O/FP/F \\
\hline $0-1$ & 54 & $51(94.4 \%)$ & $34(63 \%)$ & $48 / 45 / 30 / 3$ & $6 / 6 / 3 /-$ \\
\hline $1-2$ & 30 & $30(100 \%)$ & $24(80 \%)$ & $28 / 28 / 23 /-$ & $2 / 2 / 1 /-$ \\
\hline $2-3$ & 22 & $22(100 \%)$ & $20(90.9 \%)$ & $21 / 21 / 20 /-$ & $1 / 1 / 0 /-$ \\
\hline $3-4$ & 18 & $18(100 \%)$ & $15(83.3 \%)$ & $18 / 18 / 15 / 0$ & $0 / 0 / 0 / 0$ \\
\hline $4-5$ & 8 & $8(100 \%)$ & $7(87.5 \%)$ & $7 / 7 / 6 /-$ & $1 / 1 / 1 /-$ \\
\hline $5-6$ & 10 & $10(100 \%)$ & $8(80 \%)$ & $7 / 7 / 6 /-$ & $3 / 3 / 2 /-$ \\
\hline $6-7$ & 9 & $9(100 \%)$ & $6(66.7 \%)$ & $6 / 6 / 5 /-$ & $3 / 3 / 1 /-$ \\
\hline $7-8$ & 4 & $4(100 \%)$ & $2(50 \%)$ & $2 / 2 / 1 /-$ & $2 / 2 / 1 /-$ \\
\hline $8-9$ & 9 & $9(100 \%)$ & $6(66.7 \%)$ & $9 / 9 / 6 /-$ & $0 / 0 / 0 / 0$ \\
\hline $9-18$ & 92 & $91(98.9 \%)$ & $86(93.5 \%)$ & $79 / 78 / 78 / 1$ & $14 / 14 / 13 /-$ \\
\hline Unknown & 17 & $17(100 \%)$ & $12(70.6 \%)$ & $15 / 15 / 11 /-$ & $2 / 2 / 1 /-$ \\
\hline
\end{tabular}

Table 4: Intubation with or without assistance of medication.

\begin{tabular}{|l|l|l|}
\hline Intubations & $\begin{array}{l}\text { Assisted by } \\
\text { medication }\end{array}$ & $\begin{array}{l}\text { non medication } \\
\text { assisted }\end{array}$ \\
\hline Number of attempts & 174 & 99 \\
\hline Overall success rate & $174(100 \%)$ & $95(96 \%)$ \\
\hline First pass success rate & $154(88.5 \%)$ & $67(67.7 \%)$ \\
\hline
\end{tabular}

Table 5: Overall and first pass success rate by intubator.

\begin{tabular}{|l|l|l|}
\hline & Anesthesiologist & Trauma Surgeon \\
\hline Number of attempts & 238 & 35 \\
\hline Overall success rate & $234(98.9 \%)$ & $35(100 \%)$ \\
\hline First pass success rate & $197(82.8 \%)$ & $24(68.6 \%)$ \\
\hline
\end{tabular}

rates when the patient was intubated in a classic manner or a video-assisted device (McGrath video laryngoscope) was used (Table 2). Table 3 shows an overview of the patient's ages in this study population. Fifty-four (20\%) children under the age of 1-year were intubated.

ETI assisted by medication (at least an induction with a hypnoticum (Etomidate, Propofol or Ketamine) in combination with a muscle relaxant (Rocuronium or Suxamethonium), sometimes an opioid was added (Fentanyl)) had a significantly higher first attempt rate than ETI without medication (during CPR) (88.5\% vs. $67.7 \%, p=0.0002$ ) (Table 4). Anaesthesiologists intubated 238 paediatric patients; whereas, trauma surgeons intubated 35 paediatric patients. For the whole group together, there was no significant difference in success rates when intubated by an anaesthesiologist vs. a trauma surgeon $(p=0.09)$ (Table 5$)$. When examining intubations with and without medicine, the anaesthesiologist had a significantly better success rate; medication-assisted first attempts: $95 \% \mathrm{Cl}$ (2.8485 to 39.2547) $p=0.0133$.

In four (1.5\%) paediatric patients, ETI failed. All four were performed by an anaesthesiologist. Two failed because the vocal cords could not be visualized. The other two cases had trismus of the jaw; one of these cases surgical airway was performed. The reasons why more than one attempt was needed were: Not been able to visualize vocal cords (18), view obscured by vomit (5) or blood (1), tube size too big (2) or too small (uncuffed tube) (1), laryngoscope blade malfunction (1), difficult intubation due to facial trauma (1), corpus alienum (1), or unknown (22). The overall success rate was $100 \%$, except in the age groups: Zero to one year of age and 15 to 16 -years of age. The first attempt success rate was lower in younger children (Table 3 ) and in paediatric patients in cardiopulmonary arrest (the non-medication group). No unrecognized oesophageal intubations were discovered at admission to the hospital. 
Citation: Moors XRJ, Oude Alink MB, Bouman SJM, et al. (2019) Retrospective Analysis of out-of-Hospital Paediatric Intubation by a Dutch Helicopter Emergency Medical Service. Clin Pediatr Res 3(1):56-62

\section{Discussion}

Several studies have examined prehospital paediatric ETI performed by paramedics or emergency physicians from different clinical backgrounds, but little is known about children who undergo prehospital ETI by anaesthesiologists or anaesthesia-trained trauma surgeons [3-6,13-18,24]. Looking at the whole group, we could not find a significant difference when intubated by an anaesthesiologist or an anaesthesia-trained trauma surgeon. This is likely because the number of intubations was not large enough in the second group. Only two of a total of 11 physicians in the Rotterdam HEMS are trauma surgeons. The two trauma surgeons only intubated 35 paediatric patients, but if divided proportionally they should have intubated 50 . This could be because they might have a higher threshold for performing paediatric intubations and may try to get to the hospital earlier to avoid intubating these patients in a prehospital setting. When taking a closer look at medication-assisted intubation, a significant difference was found in favour of the anaesthesiologist; medication assisted $1^{\text {st }}$ pass: $95 \% \mathrm{Cl}$ (2.8485 to 39.2547$) \mathrm{p}=0.0133$.

Compared with the study by Hansen, et al. [12], our analysis showed a higher rate of successful first attempts ( $81 \%$ vs. $68.9 \%)$ and a higher rate of overall success (98.5\% vs. $81.1 \%$ ). Our study had a comparable overall success rate $(98.5 \%$ vs. $98.6 \%)$ to Schmidt, et al. [22], who examined highly trained physicians who intubated paediatric patients. Our success rate is comparable to in-hospital paediatric emergency ETI [25]. Like in other studies, the group of paediatric patients under the age of one year and the group of paediatric patients intubated under cardiopulmonary arrest (non-medication group) had lower first attempt rates in our study. If the group of paediatric patients under the age of one year was removed, the first attempt success rate would be $85 \%$ which is in line with other studies on physician-performed paediatric intubations. If we removed the intubations done by trauma surgeons, the first attempt success rate would be $92 \%$, which is in line with the study done by Schmidt, et al. [22], looking at highly trained physicians (92\% vs. 95\%). As stated earlier, we could not find a significant difference between the intubations performed by an anaesthesiologist vs. a trauma surgeon, but this may be due to limited numbers of patients.

In the prehospital setting, tracheal intubation is more difficult and can be challenging than when performed in hospital under controlled conditions [4-7]. This is why alternative airway devices, such as laryngeal tubes or laryngeal mask airways, have particular value for prehospital use because they require less training and have a high success rate with low incidence of complications. In the Netherlands, a previous study by Moors, et al. [15] showed that paediatric intubation by an EMS team has a high rate of failure and complications. It also showed that the overall success rate of ETI by EMS was $57 \%$, which is in the lower range compared with other studies [12]. Thus, ETI should not be performed by EMS in The Netherlands. But tracheal (paediatric) intubation offers unparalleled advantages such as uninterrupted chest compressions during cardiopulmonary resuscitation, facilitation of invasive ventilation with monitoring of end tidal $\mathrm{CO}_{2}$, high inspiratory airway pressures with or without positive end expiratory pressure (PEEP) [26] indicating that the threshold for deployment of a physician-staffed HEMS in The Netherlands should be low for paediatric patients with a compromised airway.

Of all 1905 paediatric calls, 361 (19\%) paediatric patients were intubated. This large number of paediatric intubation means that HEMS are frequently called to critically ill or severely injured children who require securing of their airway. As stated earlier, success rates are generally lower for out-of-hospital and even in-hospital paediatric intubation due to lack of training or experience with paediatric patients and paediatric intubations $[14,15,20]$. Successful airway management requires training, skills, and ongoing experience to consistently perform these procedures in an effective, timely, and safe manner [12]. Our results from the Rotterdam HEMS indicate their success rate is high, and rates were similar for the adult population and in-hospital emergency paediatric ETI [25], indicating that this is a critical but safe procedure when performed by the HEMS physician. This could be due to their frequent training and exposure to critically ill and severely injured paediatric patients.

\section{Limitations}

This was a single centre retrospective study which is a major limitation. Furthermore, essential parts of our evaluation relied on self-assessment. Therefore, we could not rule out potential bias associated with under-or-over-rating as well as under-or-over-reporting of events. However, only seven patients were excluded due to missing data.

\section{Conclusion}

Prehospital intubation of critical injured or ill paediatric patients has a high success rate when performed by HEMS physicians in The Netherlands. This emphasizes the additional value of the physician-staffed HEMS and that the threshold for deployment of HEMS should be low in cases of a potentially compromised airway in paediatric patients. The first attempt and overall success rates were significantly higher for Dutch HEMS physicians compared with ambulance paramedics in the Dutch prehospital setting. Thus, we advise that Dutch ambulance paramedics should be cautious about intubating paediatric patients without HEMS supervision.

\section{Conflict of Interest Statement}

The authors have no conflict of interest to disclose.

\section{Funding}

No funding was received.

\section{Article Summary}

\section{Why is this topic important?}

Little is known about the success rate and complications of paediatric endotracheal intubation in a prehospital setting performed by a Helicopter Emergency Medical Service (HEMS) and poor performances are seen for all providers 
Citation: Moors XRJ, Oude Alink MB, Bouman SJM, et al. (2019) Retrospective Analysis of out-of-Hospital Paediatric Intubation by a Dutch Helicopter Emergency Medical Service. Clin Pediatr Res 3(1):56-62

of emergency medical care due to a lack of frequent exposure to paediatric patients and paediatric intubations.

\section{What does this study attempt to show?}

The primary endpoints of this study were the first attempt and overall success rates for out-of-hospital, at the scene paediatric endotracheal intubation by physician-led HEMS. Secondary endpoints were the type of intubation (direct laryngoscopy or video-assisted intubation using the McGrath video laryngoscope); complications of airway management, including failure of intubation; success by operator type; patient's age, and medication-assisted intubation.

\section{What are the key findings?}

Prehospital intubation of critical injured or ill paediatric patients has a high success rate when performed by HEMS physicians in The Netherlands. The overall success rate was $98.5 \%$ and the first attempt success rate was $81 \%$. ETI assisted by medication had a significantly higher first attempt rate than ETI without medication ( $88.5 \%$ vs. $67.7 \%$, $p=0.0002$ ). When examining intubations with and without medicine, the anaesthesiologist had a significantly better success rate; medication-assisted first attempts: $95 \% \mathrm{Cl}$ (2.8485 to 39.2547) $p=0.0133$.

\section{How is patient care impacted?}

This emphasizes the additional value of the physician-staffed HEMS and that the threshold for deployment of HEMS should be low in cases of a potentially compromised airway in paediatric patients. The first attempt and overall success rates were significantly higher for Dutch HEMS physicians compared with ambulance paramedics in the Dutch prehospital setting. Thus, we advise that Dutch ambulance paramedics should be cautious about intubating paediatric patients without HEMS supervision.

\section{References}

1. Thim T, Krarup NHV, Grove EL, et al. (2012) Initial assessment and treatment with the airway, breathing, circulation, disability, exposure (ABCDE) approach. Int J Gen Med 5: 117-121.

2. Ian K Maconochie, Robert Bingham, Christoph Eich, et al. (2015) European resuscitation council guidelines for resuscitation 2015: Section 6 Paediatric Life Support. Resuscitation 95: 223-248.

3. Deakin CD, Peters R, Tomlinson P, et al. (2005) Securing the prehospital airway: A comparison of laryngeal mask insertion and endotracheal intubation by UK paramedics. Emerg Med J 22: 6467.

4. Eich C, Roessler M, Nemeth M, et al. (2009) Characteristics and outcome of prehospital paediatric tracheal intubation attended by anaesthesia-trained emergency physicians. Resuscitation 80: 1371-1377.

5. Sunde GA, Heltne JK, Lockey D, et al. (2015) Airway management by physician-staffed helicopter emergency medical services - a prospective, multicentre, observational study of 2,327 patients. Scand J Trauma Resusc Emerg Med 23: 57.

6. Burns BJ, Watterson JB, Ware S, et al. (2017) Analysis of out-of-hospital pediatric intubation by an australian helicopter emergency medical service. Ann Emerg Med 70: 773-782.
7. Timmermann A, Eich C, Russo SG, et al. (2006) Prehospital airway management: A prospective evaluation of anaesthesia trained emergency physicians. Resuscitation 70: 179-185.

8. Kamiutsuri K, Okutani R, Kozawa S (2013) Analysis of prehospital endotracheal intubation performed by emergency physicians: Retrospective survey of a single emergency medical center in Japan. J Anesth 27: 374-379.

9. Fogg T, Annesley N, Hitos K, et al. (2012) Prospective observational study of the practice of endotracheal intubation in the emergency department of a tertiary hospital in sydney, Australia. Emerg Med Australas 24: 617-624.

10. Thoeni N, Piegeler T, Brueesch M, et al. (2015) Incidence of difficult airway situations during prehospital airway management by emergency physicians - a retrospective analysis of 692 consecutive patients. Resuscitation 90: 42-45.

11. Breckwoldt J, Klemstein S, Brunne B, et al. (2011) Difficult prehospital endotracheal intubation-predisposing factors in a physician based EMS. Resuscitation 82: 1519-1524.

12. Hansen M, Lambert W, Guise JM, et al. (2015) Out-of-hospital pediatric airway management in the united states. Resuscitation 90: 104-110.

13. Bosch J, de Nooij J, de Visser M, et al. (2014) Prehospital use in emergency patients of a laryngeal mask airway by ambulance paramedics is a safe and effective alternative for tracheal intubation. Emerg Med J 31: 750-753.

14. Gerritse BM, Draaisma JM, Schalwijk A, et al. (2008) Should EMS-paramedics perform pediatric tracheal intubation in the field? Resuscitation 79: 225-229.

15. Moors XRJ, Rijs K, Den Hartog D, et al. (2018) Pediatric out-of-hospital cardiopulmonary resuscitation by helicopter emergency medical service, does it has added value compared to regular emergency medical service? Eur J Trauma Emerg Surg 44: 407410.

16. Timmermann A, Russo SG, Eich C, et al. (2007) The out-of-hospital esophageal and endobronchial intubations performed by emergency physicians. Anesth Analg 104: 619-623.

17. Tarpgaard M, Hansen TM, Rognas L (2015) Anaesthesist-provided prehospital advanced airway management in children: a descriptive study. Scand J Trauma Resusc Emerg Med 23: 61.

18. Nevin DG, Green SJ, Weaver AE, et al. (2014) An observation study of paediatric pre-hospital intubation and anaesthesia in 1933 children attended by a physician-led, pre-hospital trauma service. Resuscitation 85: 189-195.

19. Gausche M, Lewis RJ, Stratton, et al. (2000) Effect of Out-of-hospital pediatric endotracheal intubation on survival and neurological outcome: A controlled clinical trial. JAMA 283: 783-790.

20. Kerry BT, Rinderknecht AS, Geis GT, et al. (2012) Rapid sequence intubation for pediatric emergency patients: Higher frequency of failed attempts and adverse effects found by video review. Ann Emerg Med 60: 251-259.

21. Youngquist ST, Henderson DP, Gausche-Hill M, et al. (2008) Paramedic self-efficacy and skill retention in pediatric airway management. Acad Emerg Med 15: 1295-1303.

22. Schmidt AR, Ulrich L, Seifert B, et al. (2016) Ease and difficulty of pre-hospital airway management in 425 paediatric patients treated by a helicopter emergency medical service: A retrospective analysis. Scand J Trauma Resusc Emerg Med 24: 22. 
Citation: Moors XRJ, Oude Alink MB, Bouman SJM, et al. (2019) Retrospective Analysis of out-of-Hospital Paediatric Intubation by a Dutch Helicopter Emergency Medical Service. Clin Pediatr Res 3(1):56-62

23. Lockey D, Crewdson K, Weaver A, et al. (2014) Observational study of the Success rates of Intubation and failed Intubation Airway Rescue Techniques in 7256 Attempted Intubations of Trauma Patients by Pre-Hospital Physicians. Br J Anaesth 113: 220-225.

24. Lossius HM, Roislien J, Lockey DJ (2012) Patient safety in pre-hospital emergency tracheal intubation: A comprehensive meta-analysis of the intubation success rates of ems providers. Crit Care 16: 24.
25. Neuhaus D, Schmitz A, Gerber A, et al. (2013) Controlled rapid sequence induction and intubation-an analysis of 1001 children. Paediatr Anaesth 23: 734-740.

26. Maconochie IK, Bingham R, Eich C, et al. (2015) European resuscitation council guidelines for resuscitation 2015: Section 6. paediatric life support. Resuscitation 95: 223-248. 